\title{
A model for the grasping analysis of an underactuated finger driven by unextensible tendon
}

\author{
Vincenzo Niola ${ }^{1 a}$, Cesare Rossi ${ }^{1}$, Sergio Savino ${ }^{1}$ \\ ${ }^{1}$ University of Naples "Federico II" Via Claudio 21, 80125, Naples ITALY
}

\begin{abstract}
A grasping analysis is presented of an underactuated finger driven by unextensible tendon. The finger itself is one of those of a mechanical prosthesis that was principally conceived as human prosthesis. After a brief description of the whole system constituted by the mechanical hand, the model to predict the behavior of the finger during the grasping is presented; then some examples of grasping are presented. The model can be useful for both the under-actuated finger design and for the prediction of the capabilities of the whole hand.
\end{abstract}

\section{Introduction}

A number of Authors have investigated on mechanical grasping devices and hand prosthesis In particular [1-6]. These devices are based on mechanical systems using a number of motors [ 7-10 ] or on selfadapting devices using a single motor [11-13] .

Among the later, the authors of this contribution developed a new mechanical hand (Federica mechanical hand) based on a self-adaptive patented scheme (patent $n$. 0001415546 and n. 102015000059873 , the latter pending) [14-18] that is briefly described in figure 1. In

the figure a prototype is shown and a CAD design showing the working principle is also reported.

The hand is composed by five fingers, shown in figure 2 , actuated by a single motor by means of a pulley system in cascade, which automatically distributes the tendon displacement to the phalanges. Each finger consists of three elements, representing respectively the distal, medial and proximal phalanges, joined by hinges, and activated by a single inelastic tendon. The grasp of an object of any shape is obtained by operating the main inelastic tendon, which, through the pulley system, distributes the force among the various fingers, and generates the closure of each finger according to the resistance offered by the object to be grasped in the contact points.

In this contribution, the authors focused themselves on the study of the grasping of the single finger in order to optimize the finger behavior during the grasping itself.

Some examples of contact between the finger phalanxes and a solid surface having a defined geometrical shape are considered.

The contact between the finger and a surface has been studied using the Simulink library called SimMechanics contact forces downloaded from the Mathworks. The library provides different blocks according to the type of surface to be treated. We focused mainly on two blocks, the "sphere to plane" and the "sphere to tube". The object of the study is the contact between the proximal phalanges, medial and distal and a sphere and, in addition, the contact between the tip of the finger shown schematically with a cylindrical surface and a sphere.

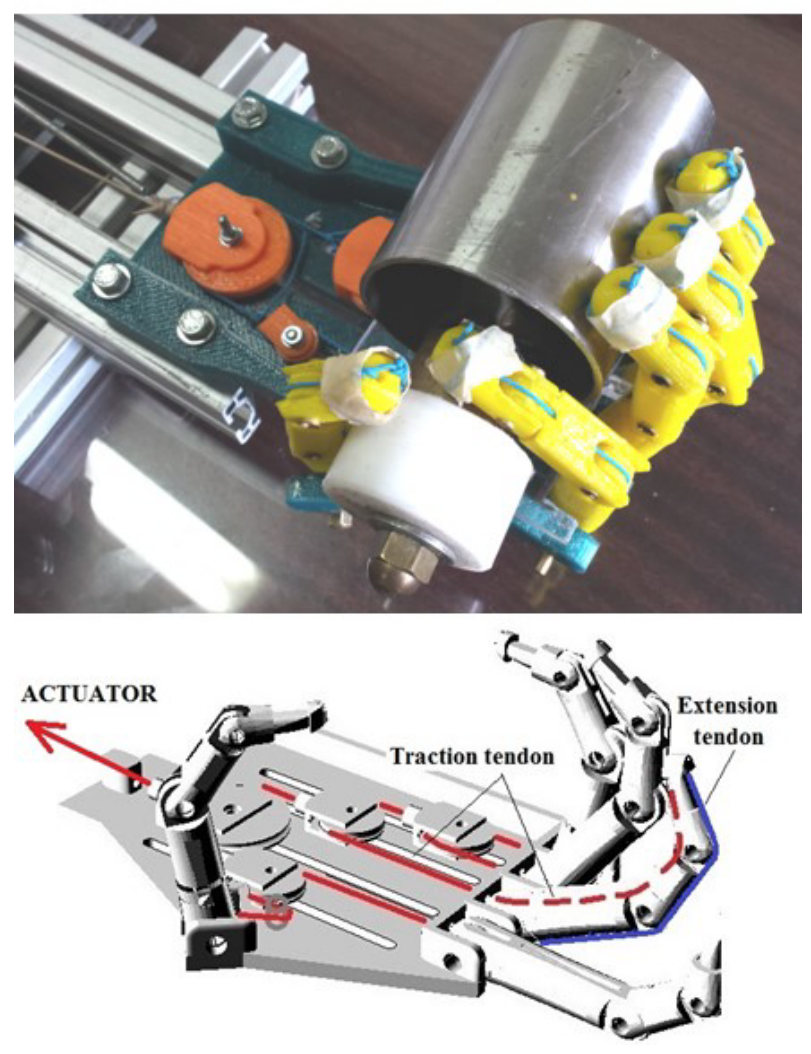

Figure 1. The underactuated, self-adapting mechanical hand.

\footnotetext{
${ }^{\mathrm{a}}$ Vincenzo Niola: vincenzo.niola@unina.it
} 

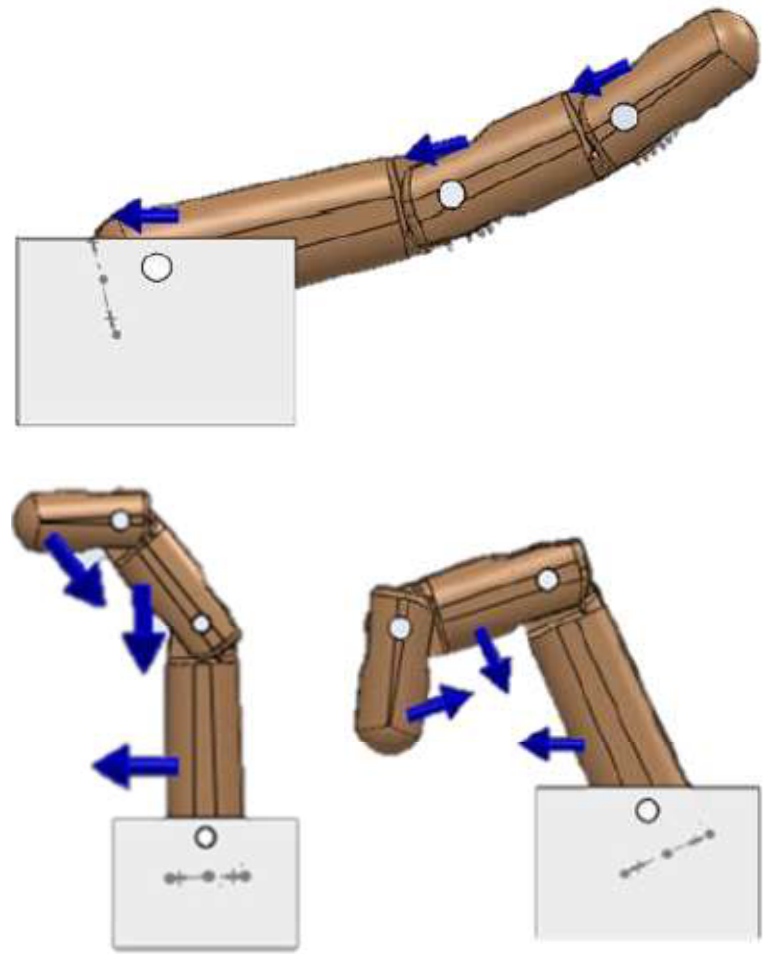

Figure 2. Scheme of the finger and closing simulation.

\section{The contact between surfaces}

The contact between the finger and a surface has been studied using the Simulink library called SimMechanics contact forces downloaded from the Mathworks. The library provides different blocks according to the type of surface to be treated. We focused mainly on two blocks, the "sphere to plane" and the "sphere to tube". The object of the study is the contact between the proximal phalanges, medial and distal and a surface and, in addition, the contact between the tip of the finger shown schematically with a cylindrical surface and a sphere.

The contact between two bodies was simulated with a force applied only along the direction of penetration of the two bodies. In figure 5 this force is shown as $F_{n}$, and it acts on the plane and on the sphere in the opposite direction. In the contact model, also the friction force is included; it acts as a tangential force in the contact point on both bodies. In figure 3 the friction force is shown as $\mathrm{F}_{\mathrm{t}}$.

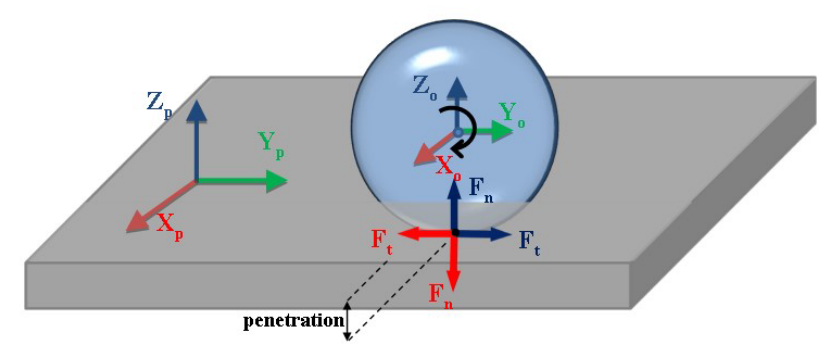

Figure 3. contact scheme with normal force Fn and friction force $F_{t}$.
The normal force $F_{n}$ acts by opposing the penetration of the two bodies and the damping is zero when the penetration decreases.

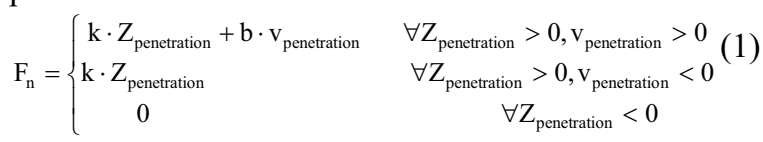

Where $Z_{\text {penetration }}$ is the penetration between the two bodies, $v_{\text {penetration }}$ is the velocity of penetration, $\mathrm{k}$ is the contact stiffness and $\mathrm{b}$ is the contact damping.

The friction force Ft is the product of normal force and a coefficient of friction that is a function of the relative velocity at the contact point.

Examples of the tool to study the contact between a sphere and a plane or between a sphere and a tube are reported in fig. 4.

By using the original blocks of SimMechanics contact forces library we observed that it is not possible to model the edge effect between finger and ball. This circumstance is a drawback because many contact intermediate positions, i.e. those in which the ball is located between two fingers, are not correctly handled (a finger can penetrate the sphere).

In order to make the appropriate corrections we considered a simpler system constituted by a plane and a sphere; in fig. 5 mentioned drawback can be observed.
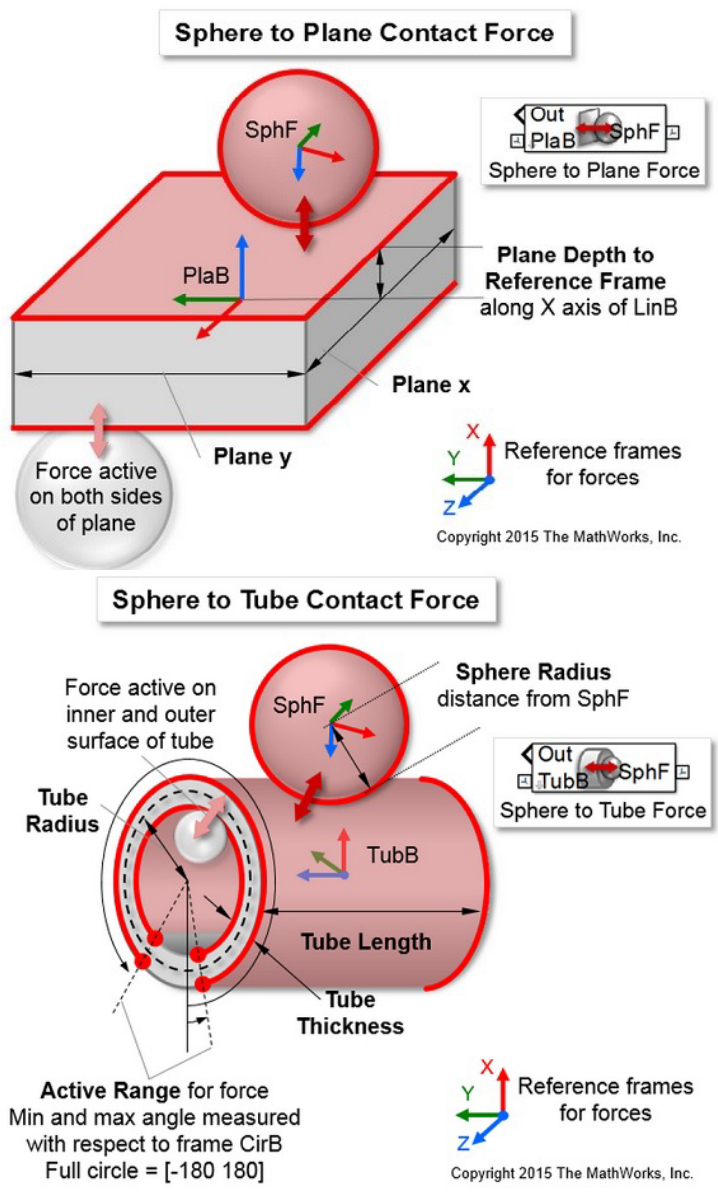

Figure 4. Contact between a sphere and a plane or between a sphere and a tube 


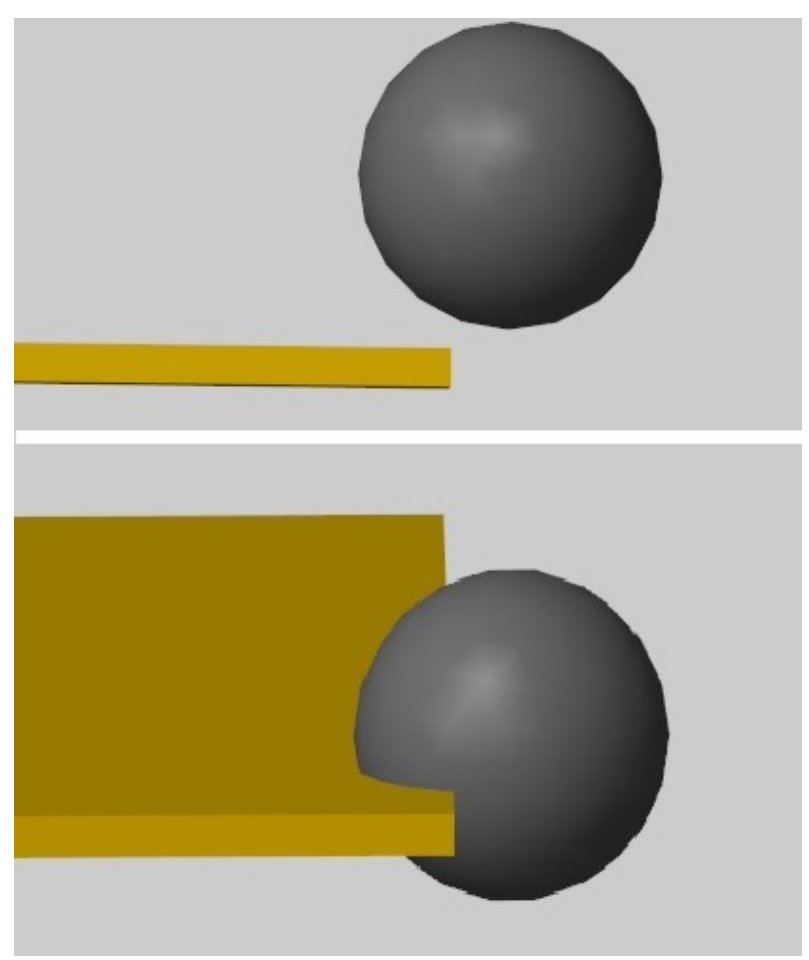

Figure5. Contact model without the "edge effect".

The main changes made to take account of the contact between the ball and the edge of the plan include the evaluation of the contact condition by introducing the following quantities:

$d x_{c}=|x|-\frac{\text { plane_length } x}{2}$
$d y_{c}=|y|-\frac{\text { plane_lengt }}{2}$
$r_{u}=\sqrt{(\text { sphere_rad })^{2}-\left(d x_{c}^{2}+d y_{c}^{2}\right)}$

Representing the distance of the sphere center from the edge of the plane, like it is possible to see in figure 6 .

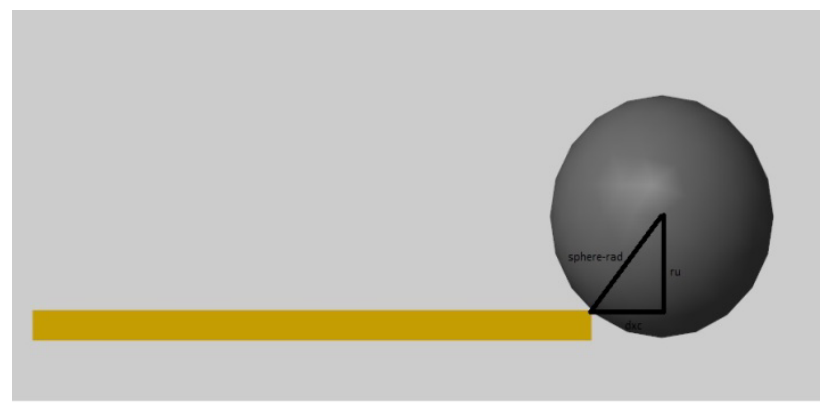

Figure 6. Point of contact of sphere when its center is out of plane.

Calculating the forces acting on the ball we reported the components of the normal force in the plane reference system according to the following equations:

$F_{z}=\frac{F_{n}}{\text { sphere-rad }} \cdot \sqrt{\text { sphere }- \text { rad }^{2}-d x_{c}^{2}-d y_{c}^{2}}$
$F_{x}=\frac{\sqrt{F_{n}^{2}-F_{z}^{2}}}{\sqrt{d x_{c}^{2}+d_{c}^{2}}} \cdot d x_{c}$

$F_{y}=\frac{\sqrt{F_{n}^{2}-F_{z}^{2}}}{\sqrt{d x_{c}^{2}+d y_{c}^{2}}} \cdot d y_{c}$

This in order to permit a correct impact between ball and plane and an eventual fall from the plane.

The sign of the moments of the forces acting on the sphere was changed in order to permit the correct rotation (clockwise or counter clockwise) depending on the contact of the sphere with the edge of the plane.

Similar expedients were adopted for the contact between ball and tube. In Figure 7 a wrong contact (up) and a wrong contact between ball and tube are shown.
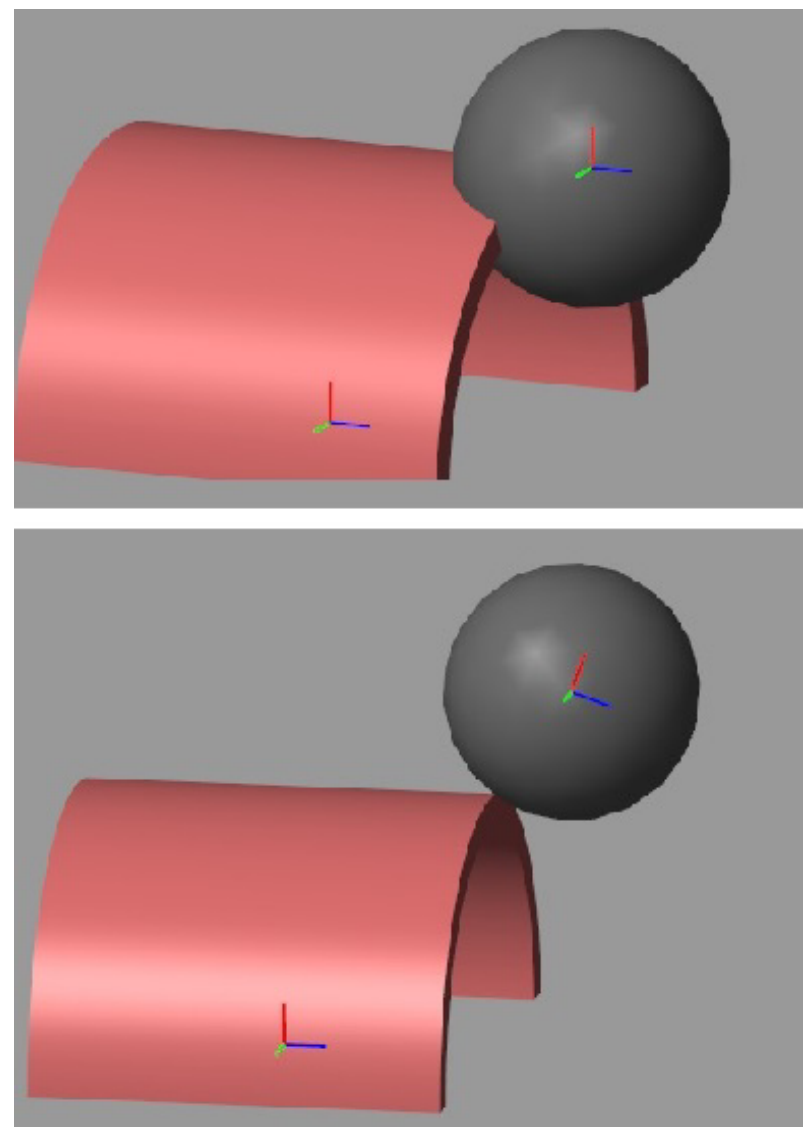

Figure 7. Contact model between sphere and tube without the "edge effect", and with "edge effect".

\section{Simulation results}

In this section some of the simulation results are reported.

\subsection{The contact between finger and sphere}

The blocks of the library SimMechanics Contact Forces, originally forecast in the model Federica hand, involve a situation like the one shown in figure 8 , where penetration occurs between finger and ball. 


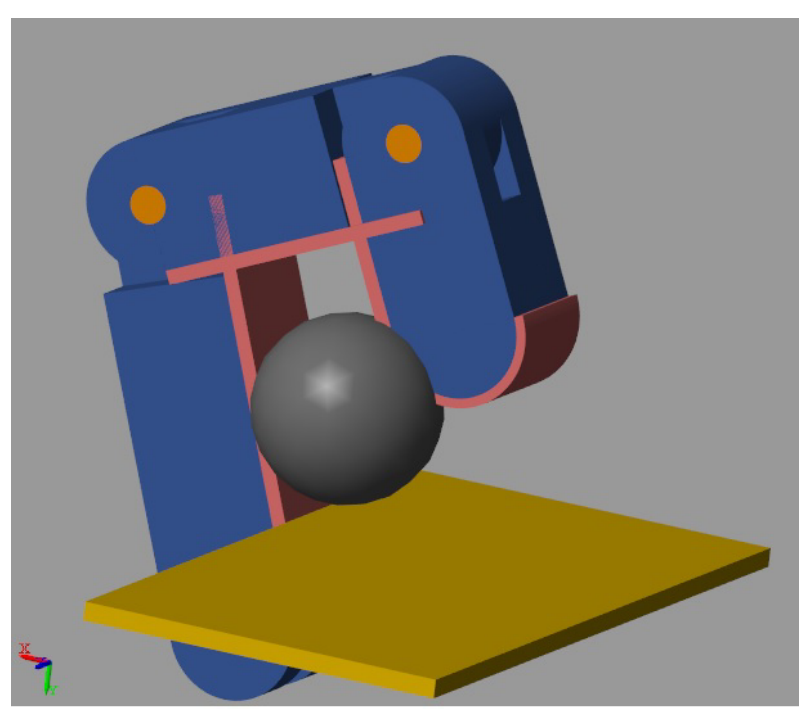

Figure 8. Finger in contact with sphere without "edge effect"

Thanks to the changes seen in the previous paragraphs, it is possible to evaluate all the intermediate positions; an example of this result is shown in figure 9.

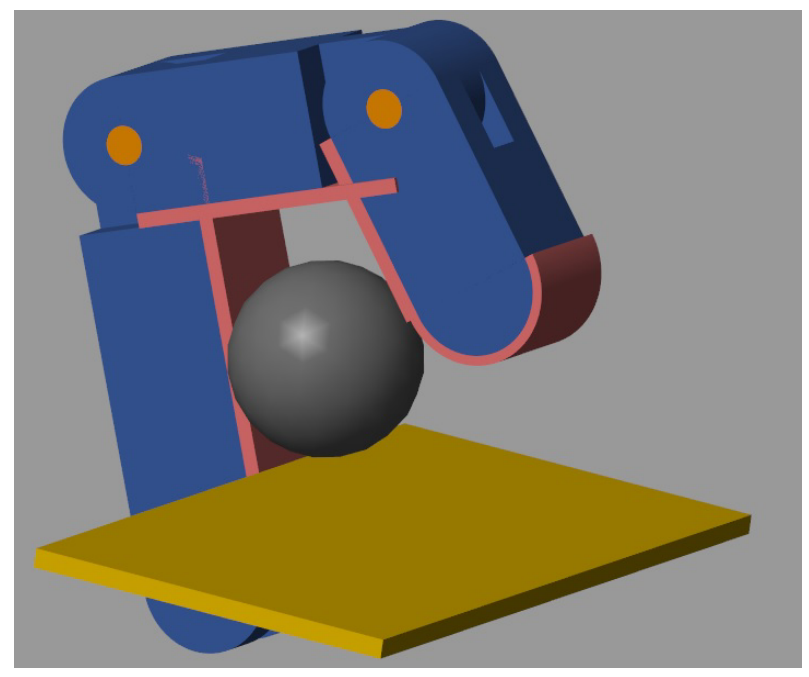

Figure 9. Finger in contact with sphere with "edge effect"

For each of the fingers, four blocks sphere to plane were used in order to simulate the contact between the inner surfaces of the phalanxes and the sphere and between the palm of the hand and the sphere and another block sphere to tube for the contact between the fingertip and the sphere.

In the simulations we assumed to grab spheres having different sizes that can be fixed on the palm in a specific position (using the weld joint block of SimMechanics) or can be free to move on the palm. The latter situation is supposed to be a result of an impact between the sphere and a finger phalanx before the sphere was grabbed; this was obtained by using the bushing joint block. The output variables of each contact block are analyzed in a subsystem called Grasp.

The grasping conditions require that a value based on the number of surfaces (i.e. the number of the phalanges) that touch the ball is assigned:

2 -> Object contact with at least 3 surfaces
1 -> Object contact with at least two surfaces

0.75 -> Object speed nothing compared to the palm

0.5 -> Object speed nothing compared to the phalanges

In figure 10 a graph showing the contact during the time is shown; the graph refers to the contact during the grasping of a sphere having unitary radius. The fluctuating trend in the graph is due to the fact that rebounds may occur due to elastic forces.

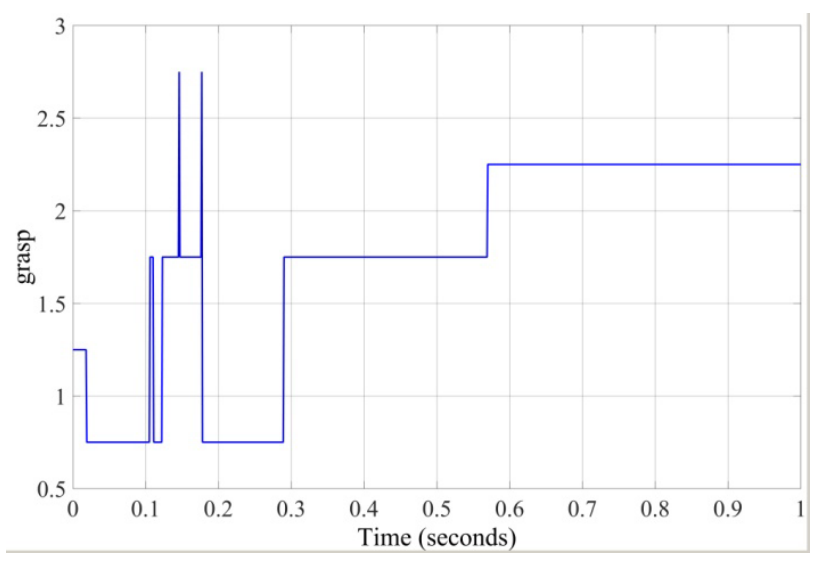

Figure 10. Grasp measure during a grasping task.

\section{Conclusion}

A model to analyze the grasping of an underactuated finger driven by unextensible tendon was presented. The finger itself is one of those of a mechanical prosthesis that was principally conceived as human prosthesis.

The reported examples seems to indicate that the model can predict the behavior of the finger during the grasping.

The model can be useful for both the under-actuated finger design and for the prediction of the capabilities of the whole hand. Also the actuator's optimum law of motion, computed by means of a previously proposed algorithm, [19], can represent a field of study that can be improved using the presented model.

Moreover, the model allows measuring the rotations of each phalanx, allowing to test the measurements carried out on real prototypes, by means of previously developed vision systems, [20-23].

The proposed method is also suitable to study several different topics (even very different from this one) the authors are investigating on. Among these, even the dynamics of the throwing machines (see e.g. [25-27]), as for the impact between their component is concerned can be studied.

\section{ACKNOWLEDGEMENT}

During this research a valuable help was provided by Mr. Francesco Iodice who was working for the bachelor's degree. The authors thank him for his diligence and his help. 


\section{References}

1. Suárez, R., Roa, M. A., \& Cornellà, J., Grasp quality measures. Technical University of Catalunya, Technical Report IOCDT-P-2006-10 (2006)

2. Roa, M. A., Suárez, R., Grasp quality measures: review and performance, Autonomous Robots, vol 38, Issue 1, pp 65-88 (2015)

3. Gabiccini, M., Farnioli, E., and Bicchi, A., Grasp and manipulation analysis for synergistic underactuated hands under general loading conditions, In International Conference of Ro-botics and Automation - ICRA 2012, pages 2836 - 2842, Saint Paul, MN, USA (2012).

4. Gabiccini, M., Farnioli, E., Bicchi, A., Grasp Analysis Tools for Synergistic Underactuated Robotic Hands. International Journal of Robotic Research, vol. 32, pp. 1553-1576 (2013)

5. Lotti, F., Vasura, G., Design aspects for advanced robot hands, In Proc. of IEEE/RSJ In-ternational Conference on Intelligent Robots and Systems, Lausanne, Switzerland, sep-tember 30 - October 4, 2002.

6. Roccella, S., Carrozza, M.C., Cappiello, G., Dario, P., Cabibihan, J.J., Zecca, M., Miwa, H., Itoh, K., Matsumoto, M., Takanishi, A., Design, fabrication and preliminary results of a Novel anthropomorphic hand for humanoid robotics: $\mathrm{RCH}-1$, Proceedings of 2004 IEEE/RSJ International Conference on Intelligent Robots and Systems, Sendai, Japan, September 28 - October 2, 2004

7. Hirose, S., Umetani, Y, The development of soft gripper for versatile robot hand, Mecha-nism and Machine Theory, vol. 13, pp. 351-359 (1978)

8. Catalano M. Grioli, G., Serio, A. , Farnioli, E., Piazza, C. and Bicchi, A., Adaptive Syner-gies for a Humanoid Robot Hand. In Proc. of IEEE-RAS International Conference on Hu-manoid Robots, Osaka (Japan), October 2012

9. Townsend, W., The BarretHand grasper programmably flexible part handling and assem-bly, Industrial Robot: An International Journal, vol.27, no. 3, pp. 181-188 (2000)

10. Baril M., Laliberte T., Gosselin C., Routhier F., On the Design of a Mechanically Pro-grammable Underactuated Anthropo-morphic Prosthetic Gripper. Journal of Mechanical Design, issue 135, vol 12, doi:10.1115/1.4025493 (2013)

11. Dechev N., Cleghorn, W.L., Naumann, S., Multiple finger, passive adaptive grasp prosthetic hand, Mechanism and Machine Theory, vol. 36, pp 11571173 (2001)

12. Gosselin C., Pelletier, F., and Lalibertè, T. An Anthropomorphic Underactuated Robotic Hand with 15 Dofs and a Single Actuator. In Proc. of 2008 IEEE International Conference on Robotics and Automation, Pasadena (CA, USA), May 19-23 (2008)

13. Groenewegen, M. W., Aguirre, M. E., \& Herder, J. L., Design of a Partially Compliant, Three-Phalanx Underactuated Prosthetic Finger. In ASME 2015 International Design Engineering Technical
Conferences and Computers and Information in Engineering Conference American Society of Mechanical Engineers (2015)

14. Rossi, C., Savino, S., Mechanical Model of a Single Tendon Finger, Proc. of ICNAAM 2013: 11th International Conference of Numerical Analysis and Applied Mathematics, Rhodes, Greece, Sep 21-27 (2013)

15. Penta, F., Rossi, C., Savino, S., An Underactuated Finger for a Robotic Hand. International Journal of Mechanics and Control, Vol. 15, n.2, ISSN: 15908844 (2014)

16. Niola, V., Rossi, C., Savino, S. (2014). "A new mechanical hand: Theoretical studies and first prototyping", International Review of Mechanical Engineering, volume 8, issue 5, pp. 835-844, ISSN:19708734.

17. Niola, V., Penta, F., Rossi, C., Savino, S., An underactuated mechanical hand: Theoretical studies and prototyping, International Journal of Mechanics and Control, Volume 16, Issue 1, pp. 11-19 (2015)

18. Carbone G., Rossi C., Savino S., (2015). "Performance comparison between Federica Hand and LARM Hand", International Journal of Advanced Robotic Systems, 14 July 2015, Volume 12, DOI: 10.5772/60523, ISSN: 17298806

19. Niola, V., Rossi, C., Savino, S. and Strano, S., Robot trajectory planning by points and tangents. In Proceedings of the 10th WSEAS International Conference on Robotics, Control and Manufacturing Technology, ROCOM '10, Hangzhou, China, April 11-13, 2010, pp-91-96 (2010)

20. V. Niola, C. Rossi, S. Savino - "A New Real Time Shape Acquisition with a Laser Scanner: First Test Results" - ROBOTICS AND COMPUTERINTEGRATED MANUFACTURING, issue 6, vol. 26, December 2010, pp 543-550, ISSN 0736-5845.

21. V. Niola, C. Rossi, Video acquisition of a robot arm trajectories in the work space, WSEAS Transactions on Computers, vol. 4, issue 7, pp. 830-836, (2005)

22. Niola, V., Quaremba, G., Amoresano, A., A study on infrared thermography processed trough the wavelet transform, Proceedings of the 8th WSEAS International Conference on System Science and Simulation in Engineering, ICOSSSE '09, pp 57-62 (2009)

23. M. Martorelli, C. Rossi, S. Savino, G. Staiano (2015) A Contactless Robot Kinematic Calibration Method by Digital Photogrammetry. International Journal of Mechanics and Control (JoMaC) vol. 16, No.2, ISSN: 1590-8844.

24. Rossi, C. Ancient throwing machines: A method to calculate their performance, Mechanism and Machine Theory, Volume 51, Pages 1-13 (2012).

25. Rossi, C., Russo, F., A reconstruction of the GreekRoman repeating catapult, Mechanism and Machine Theory, Volume 45, Issue 1, pp 36-45 (2010)

26. Rossi, C., Pagano, S., A study on possible motors for siege towers, Journal of Mechanical Design, Transactions of the ASME, Volume 133, Issue 7, Article number 071009 (2011) 